Pacific Journal of Mathematics

A FREE BOUNDARY VALUE PROBLEM FOR CAPILLARY 


\section{A FREE BOUNDARY VALUED PROBLEM FOR CAPILLARY SURFACES}

Claus GerhardT

We consider the usual capillary variational problem with an obstacle condition given on the boundary and prove existence and regularity of the solution to this problem.

o. Introduction. The equilibrium surface in a capillary tube separating liquid and gas is represented mathematically as the graph of a function $u$ solving the variational problem

(0.1) $J(v):=\int_{\Omega}\left(1+|D v|^{2}\right)^{1 / 2}+\frac{\kappa}{2} \int_{\Omega}|v|^{2}+\int_{\partial \Omega} \beta \cdot v \longrightarrow \min \forall v \in B V(\Omega)$.

Here, $\Omega$ is the cross-section of the tube, $\kappa$ a positive constant, and $\beta$ a prescribed function satisfying

$$
|\beta| \leqq 1 \text {. }
$$

$B V(\Omega)$ is the space of functions of bounded variation, the largest function class for which the variational problem can be formulated analytically.

A regular solution of the variational problem is also a solution of the corresponding Euler-Lagrange equation

$$
A u+\kappa u=0 \text { in } \Omega
$$

satisfying the natural boundary condition

$$
-a^{i} \cdot \nu_{i}=\beta \text { on } \partial \Omega,
$$

where

$$
A u=-D_{i}\left(a^{i}(D u)\right), \quad a^{i}(p)=p^{i}\left(1+|p|^{2}\right)^{-1 / 2}
$$

is the minimal surface operator in divergence form and $\nu$ the exterior unit normal to $\partial \Omega$. We note that we use throughout the text the usual summation convention to sum over repeated indices.

The formulation of the variational problem (0.1) contains implicitly the assumption that the capillary tube has an infinite length: the rise of the liquid is not constrained by the upper end of tube. Physically, and mathematically as well, it is sufficient to assume the tube to be large enough, so that the liquid is always strictly below the upper aperture of the tube. But in certain circumstances it could happen that the liquid would like to rise higher than is permitted by the configuration of the tube. 
In those cases the variational problem (0.1) is no longer correct; the correct formulation is

$$
J(v) \longrightarrow \min \quad \forall v \in B V(\Omega) \cap\left\{\left.v\right|_{\partial \Omega} \leqq \varphi\right\},
$$

where $\varphi$ is a given function defined on $\partial \Omega$ representing the constraint.

However, it is well-known that the variational problem (0.5) cannot be solved directly since the class of competing functions is not closed with respect to weak convergence in $B V(\Omega)$. To overcome this difficulty, let us write the side-condition

$$
v \leqq \varnothing
$$

as an isoperimetric constraint, namely, in the form

$$
\int_{\partial \Omega} \max (v-\varphi, 0)=0 \text {. }
$$

Then, applying Lagrange's multiplier rule formally, we are led to

$$
J(v)+\lambda \cdot \int_{\partial \Omega} \max (v-\varphi, 0) \longrightarrow \min \quad \forall v \in B V(\Omega),
$$

where $\lambda \in \boldsymbol{R}$ is an unknown multiplier to be determined: one has to show that there exists $\lambda \in \boldsymbol{R}$, and a solution $u_{\lambda}$ of (0.8) satisfying (0.7). Then, $u_{\lambda}$ would also be a solution of (0.5).

In the next sections we shall prove this under certain natural restrictions of the data and of the boundary of $\Omega$. One of those restrictions is what we call the compatibility of $\beta$ and $\lambda: \beta \in L^{\infty}(\partial \Omega)$ and $\lambda \in[0,2)$ are said to be compatible if there exists $a>0$, s.t.

$$
\sup _{\partial \Omega}|\beta+t| \leqq(1-a) \quad \forall 0 \leqq t \leqq \lambda .
$$

In $\S 1$ we prove that the variational problem (0.8) has a solution $u_{\lambda} \in C^{2}(\Omega) \cap L^{\infty}(\Omega)$ (the solution is actually real analytic in $\Omega$ ) for any pair of compatible $\beta$ and $\lambda$. In $\S 2$ it is proved that $u_{\lambda}$ is of class $H^{1, \infty}(\Omega) \cap H^{2,2}(\Omega)$, and that the side-condition (0.6) is satisfied if, e.g., the mean curvature of $\partial \Omega$ is positive, which means in the physical case that $\Omega$ is convex, and if in addition the oscillation of $\beta$ is small, where the "smallness" depends on $\partial \Omega$ and $\phi$. Finally, in $\S 3$ we consider the corresponding capillary problem with the further side-condition of prescribed volume which in fact incorperates two side-conditions, namely the volume constraint and an obstacle condition: the liquid is bounded from below by the bottom of the vessel.

No detailed proofs are given, since everything has already been proved in previous papers (cf. $[2,3,4,6]$ ) in a rather general sett- 
ing. We only apply those results to the present problem, which has been drawn to our attention by Willi Jäger to whom we are therefore very much indebted.

1. Weak solutions of the variational problem. We consider the variational problem $(0.8)$ in a slightly more general fashion, namely, in a bounded, open set $\Omega \subset \boldsymbol{R}^{n}, n \geqq 2$, with Lipschitz boundary $\partial \Omega$, we consider the variational problem

$$
\begin{aligned}
J_{\lambda}(v): & =\int_{\Omega}\left(1+|D v|^{\mid 2}\right)^{1 / 2}+\int_{\Omega} \int_{0}^{v} H(x, t) \\
& +\int_{\partial \Omega} \beta \cdot v+\lambda \cdot \int_{\partial \Omega} \max (v-\rho, 0) \longrightarrow \min \forall v \in B V(\Omega),
\end{aligned}
$$

where $H=H(x, t)$ is Lipschitz continuous satisfying

$$
\frac{\partial H}{\partial t} \geqq \kappa>0,
$$

where $\varphi$ and $\beta$ are given measurable functions on $\partial \Omega$ with

$$
\varphi \in L^{1}(\partial \Omega)
$$

and $\lambda \in[0,2)$ is a fixed multiplier, such that $\beta$ and $\lambda$ are compatible. We may write the boundary integrals in abbreviated form as

$$
\int_{\partial \Omega} j_{\lambda}(x, v-\varphi)
$$

where

$$
j_{\lambda}(x, t):=\beta \cdot t+\lambda \max (t, 0)+\beta \cdot \varphi .
$$

$j_{2}$ is measurable in $x$, and convex and Lipschitz continuous in $t$ with

$$
\left|j_{\lambda}(x, s)-j_{\lambda}(x, t)\right| \leqq(1-a) \cdot|t-s|
$$

and

$$
j_{\lambda}(\cdot,-\phi) \in L^{1}(\partial \Omega) .
$$

This can most easily be seen by looking at the 'subdifferential $\partial j_{\lambda}$ of $j_{i}$ with respect to $t$

$$
\partial j_{\lambda}(\cdot, t)=\beta+\lambda \beta_{0}(t),
$$

where

$$
\beta_{0}(t):=\left\{\begin{array}{cc}
0, & t<0 \\
{[0,1],} & t=0 \\
1, & t>0
\end{array}\right.
$$


hence the result in view of the compatibility of $\beta$ and $\lambda$.

If we assume that $\Omega$ satisfies a so-called internal sphere condition (ISC), i.e., if we assume that every point $x \in \Omega$ is contained in a ball $B$ of fixed radius $R$ lying entirely in $\Omega$, then the following theorem has been proved in [4; Theorems 2.1 and 2.2], ${ }^{1}$ see also [2]:

THEOREM 1.1. Under the assumptions stated above the variational problem

$$
\begin{aligned}
J_{\lambda}(v):= & \int_{\Omega}\left(1+|D v|^{2}\right)^{1 / 2}+\int_{\Omega} \int_{0}^{v} H(x, t) \\
& +\int_{\partial \Omega} j_{\lambda}(x, v-\varphi) \longrightarrow \min \forall v \in B V(\Omega)
\end{aligned}
$$

has a unique solution $u_{2}$ satisfying moreover

$$
u_{\lambda} \in C^{2, \alpha}(\Omega) \cap L^{\infty}(\Omega)
$$

for any $\alpha \in(0,1)$.

The proof is based on the following inequality (cf. [2; Remark 2])

$$
\int_{\partial \Omega}|v| \leqq \int_{\Omega_{\varepsilon}}|D v|+c_{\varepsilon} \int_{\Omega}|v| \quad \forall v \in B V(\Omega)
$$

where

$$
\Omega_{\varepsilon}:=\{x \in \Omega: \operatorname{dist}(x, \partial \Omega)<\varepsilon\},
$$

from which one can immediately deduce that a minimizing sequence of the variational problem (1.9) is uniformly bounded in $B V(\Omega)$, and that the functional $J_{\lambda}$ is lower semicontinuous with respect to weak convergence in $B V(\Omega)$ (cf. [2; Appendix II] and [4; Theorem 2.1]). The regularity result then follows from the minimizing property of the solution and from the interior gradient estimates for nonparametric surfaces of prescribed mean curvature (see e.g., [5]).

We note that, using the a priori estimates of Concus and Finn [1], we may conclude that the results of Theorem 1.1 are still valid in the limiting case where $a=0$ (cf. [4; Theorem 4.1]).

2. Global regularity and boundary behavior of a solution. Besides the conditions of the preceding section we assume now that $\partial \Omega \in C^{3}, \varphi \in C^{2}(\partial \Omega)$ (for simplicity), and $\beta \in C^{0,1}(\partial \Omega)$. Let us consider the corresponding boundary value problem to the variational problem (1.9)

${ }^{1}$ In that paper we only treat the case $\varphi=0$, but the results and proofs are also valid in the general case. 


$$
\begin{aligned}
A u_{\lambda}+ & H\left(x, u_{\lambda}\right)=0 \\
& -a^{i} \cdot \nu_{i} \in \beta+\lambda \beta_{0}\left(u_{\lambda}-\varphi\right) .
\end{aligned}
$$

The following theorem has been proved in [6; Theorem 3.1].

THEOREM 2.1. The boundary value problem (2.1) has for compatible $\beta$ and $\lambda$ a unique solution $u_{\lambda} \in H^{1, \infty}(\Omega) \cap H^{2,2}(\Omega)$.

The proof is based on the tangential gradient estimate of Simon and Spruck [8] for capillary surfaces, which can be applied to the present situation in view of the monotonicity of $\beta_{0}$.

Evidently, the solution of the boundary value problem is also a solution of the variational problem, and vice versa. To conclude that $u_{\lambda}$ is a solution of the original constrained problem for a suitable $\lambda$, we have to show

$$
u_{\lambda} \leqq \varphi \quad \text { on } \partial \Omega \text {. }
$$

To prove this boundary estimate we assume moreover that

$$
-H(x, \varphi) \leqq(n-1) \cdot H_{n-1}(x) \quad \forall x \in \partial \Omega,
$$

where $H_{n-1}$ is the mean curvature of $\partial \Omega$, and that

$$
\underset{\partial \Omega}{\operatorname{osc}} \beta \leqq \delta
$$

where $\delta$ is a small positive constant depending on $\partial \Omega$ and $\varphi$.

With these assumptions we can prove

THEOREM 2.2. The solution $u_{\lambda}$ of the boundary value problem (2.1) satisfies (2.2). The inequality also holds only locally, if the conditions (2.3) and (2.4) are only locally fulfilled.

The proof of the theorem can be found in [6; $\S \S 4$ and 5]; it is based on the construction of an appropriate barrier function $\delta^{+} \epsilon$ $C^{2}\left(\bar{\Omega}_{\varepsilon}\right)$ satisfying

$$
\begin{array}{ll}
A \delta^{+}+H\left(x, \delta^{+}\right) \geqq 0 & \text { in } \Omega_{\varepsilon}, \\
u \leqq \delta^{+} & \text {on } \Gamma_{\varepsilon}, \\
\varphi \leqq \delta^{+} & \text {on } \partial \Omega,
\end{array}
$$

and

$$
-a^{i}\left(D \delta^{+}\right) \cdot \nu_{i} \leqq \beta+\lambda \text { on } \partial \Omega,
$$

where $\Omega_{\varepsilon}=\{x \in \Omega: \operatorname{dist}(x, \partial \Omega)<\varepsilon\}$ and

$$
\Gamma_{\varepsilon}=\{x \in \Omega: \operatorname{dist}(x, \partial \Omega)=\varepsilon\} .
$$


The construction of $\delta^{+}$satisfying (2.5)-(2.7) is possible in view of the assumption (2.3) (cf. [7]), while the estimate (2.8) is valid if $\beta$ satisfies (2.4) and $\lambda$ is chosen appropriately, since

$$
\sup _{\partial \Omega \Omega}\left|a^{i}\left(D \hat{\delta}^{+}\right) \cdot \nu_{i}\right|<1 \text {. }
$$

Evidently, the solution of the constrained problem should be less than the solution of the unconstrained capillary problem. This is indeed the case

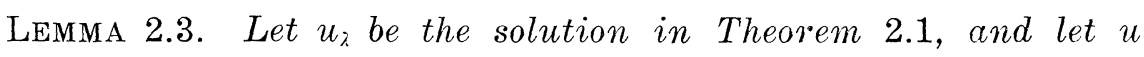
be the solution of the free capillary problem

$$
\begin{aligned}
A u+H(x, u)=0 & \text { in } \Omega, \\
-a^{i} \cdot \nu_{i}=\beta & \text { on } \partial \Omega,
\end{aligned}
$$

then

$$
u_{2} \leqq u
$$

The proof is the same as that of Theorem 2.2, since in view of (2.10)

$$
-a^{i}(D u) \cdot \nu_{i} \leqq \beta+\lambda \beta_{0}\left(u_{i}-\phi\right)
$$

on $\partial \Omega$; remember that $\beta_{0}$ is a nonnegative maximal monotone graph.

3. Solutions with prescribed volume. Let us consider liquid in a cylindrical vessel the bottom and the walls of which are made from different materials: the bottom should be such, that the liquid will not form drops on the bottom even if the volume is small, but will creep to the walls to form a usual capillary surface there.

If we look at the free boundary value problem of the previous sections in this new configuration, then the variational problem (1.9) e.g., now takes the form

$$
J_{\lambda}(v) \longrightarrow \min \quad \forall v \in B V(\Omega) \cap\{v \geqq \dot{\psi}\} \cap\left\{\int_{\Omega}(v-\psi \dot{\psi})=V\right\},
$$

where the volume $V>0$ is prescribed, and the obstacle $\psi^{\prime}$ represents the bottom.

Let us furthermore assume in this section, that $H=H(x, t)$ is sublinear in the second variable, i.e.,

$$
\sup _{\Omega} H(x, t) \leqq c \cdot(1+t) \quad \forall t>0,
$$

then we deduce from the general results in [4]. 
THEOREM 3.1. Let the conditions of Theorem 1.1 be satisfied and assume moreover $\& \in C^{0,1}(\bar{\Omega})$. Then, for any $V>0$, the variational problem (3.1) has a unique solution $u_{\lambda} \in C^{0,1}(\Omega) \cap L^{\infty}(\Omega)$. Moreover, there exists a Lagrange multiplier $\mu$, such that $u_{\lambda}$ also solves the variational problem

$$
J_{\lambda}(v)+\mu \int_{\Omega} v \longrightarrow \min \forall v \in B V(\Omega) \cap\{v \geqq \psi\} .
$$

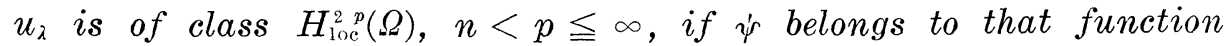
class.

We note that this result remains valid even if we only assume $H$ to satisfy the weak monotonicity condition $\partial H / \partial t \geqq 0$, i.e., even in the absence of a gravitational field. This is due to the fact, that $\left|u_{\lambda}\right|$ can be bounded independent of $\kappa$ only in terms of $V$ and $a$.

If we assume

$$
\dot{\psi} \in C^{2}(\bar{\Omega}),\left.\psi\right|_{\partial \Omega}<\varphi
$$

and

$$
\beta \leqq-a^{i}\left(D_{\psi} \dot{\gamma}\right) \cdot \nu_{i} \text { on } \partial \Omega
$$

then we obtain for coercive $H$ and for $\varphi \in C^{2}(\partial \Omega)$.

TheOREM 3.2. Suppose that the assumptions of Theorem 2.1, and (3.4), (3.5) are valid. Then the solution $u_{2}$ of the boundary value problem (3.3) is of class $H^{1, \infty}(\Omega) \cap H^{2,2}(\Omega)$ and satisfies

$$
A u_{\lambda}+H\left(x, u_{\lambda}\right)+\mu=\left\{\begin{array}{r}
0, u_{\lambda}>\psi \\
\geqq 0, u_{\lambda}=\psi
\end{array}\right.
$$

and

$$
-a^{i} \cdot \nu_{\imath} \in \beta+\lambda \beta_{0}\left(u_{\lambda}-\varphi\right) \text { on } \partial \Omega \text {. }
$$

Proof. For the proof one has to apply the ideas of $[3 ; \S 3]$ to the estimates in [6]. Let $\theta$ be the maximal monotone graph

$$
\theta(t)=\left\{\begin{aligned}
-1, & t<0 \\
{[-1,0], } & t=0 \\
0, & t>0,
\end{aligned}\right.
$$

and let $\theta_{\varepsilon}$ be a sequence of smooth monotone graphs tending to $\theta$ such that

$$
\theta_{\varepsilon}(t)=\left\{\begin{array}{c}
-1, t<-\varepsilon \\
0, t \geqq 0
\end{array}\right.
$$


Furthermore let $\mu$ be a positive constant such that

$$
A \psi+H(x, \psi) \leqq \mu \quad \text { in } \Omega .
$$

Then, we consider the approximating boundary value problems

$$
\begin{array}{r}
A u_{\lambda, \varepsilon}+H\left(x, u_{\lambda, \varepsilon}\right)+\mu \Theta_{\varepsilon}\left(u_{\lambda, \varepsilon}-\psi\right)=0 \\
-a^{i} \cdot \nu_{i} \in \beta+\lambda \beta_{0}\left(u_{\lambda, \varepsilon}-\varphi\right) .
\end{array}
$$

The estimates in [6] yield the existence of a unique solution $u_{i, \varepsilon} \in H^{1, \infty}(\Omega) \cap H^{2,2}(\Omega)$ of (3.11) with uniformly bounded norm in that function space.

From (3.4) and (3.5) we then conclude

$$
u_{\lambda, \varepsilon} \geqq \psi-\varepsilon,
$$

hence the result.

Indeed, let $\psi_{\varepsilon}:=\psi^{\prime}-\varepsilon$ and $\eta:=\max \left(\psi_{s}-u, 0\right)$. Then, combining the inequality

$$
\begin{aligned}
A \psi_{s}+H\left(x, \psi_{\varepsilon}\right)+\mu \cdot \Theta_{\varepsilon}\left(\psi_{\varepsilon}-\psi\right. & \\
\leqq & =\psi \\
& +H(x, \psi)-\mu \leqq 0
\end{aligned}
$$

with (3.11) we obtain

$$
\begin{aligned}
0 \leqq \int_{\Omega}\{ & \left.\alpha^{i}(D u)-a^{i}\left(D \psi_{\varepsilon}\right)\right\} \cdot D_{i} \eta \\
& +\int_{\Omega}\left\{H\left(x, u_{\lambda, \varepsilon}\right)-H\left(x, \psi_{\varepsilon}\right)\right\} \cdot \eta+\int_{\hat{\partial} \Omega}\left\{\beta+\lambda \beta_{0}\left(u_{\lambda, \varepsilon}-\phi\right)\right. \\
& \left.+a^{i}(D \psi) \cdot \nu_{i}\right\} \cdot \eta .
\end{aligned}
$$

The boundary integral is nonpositive on account of (3.5), since in $\{\eta>0\}$ we have $\beta_{0}\left(u_{\lambda, \varepsilon}-\varphi\right)=0$ in view of (3.4). The strict monotonicity of $H$ and the monotonicity of the vector-field $\left(a^{i}\right)$ then yield $\eta=0$.

The question, whether

$$
u_{\lambda} \leqq \varphi \quad \text { on } \partial \Omega,
$$

is not so easy to solve in the present case as in the former situation. For small volume $V$, say,

$$
V \leqq V_{0} \text {, }
$$

the inequality (3.15) is immediately verified under exactly the same conditions as in $\S 2$, where the bound $V_{0}$ depends on the given data; for large volumes the estimate (3.15) may not be valid, as is evident in the physical case.

To prove the existence of a bound $V_{0}$, let us look at the varia- 
tional problem analogous to (3.1), but without any volume constraint,

$$
J_{\lambda}(v) \longrightarrow \min \quad \forall v \in B V(\Omega) \cap\{v \geqq \psi\},
$$

where we shall always assume that $H$ is strictly monotone in the sense of condition (1.2). Let $\bar{u}_{2}$ be a solution to (3.17). We know that $\bar{u}_{2} \in H^{1, \infty}(\Omega) \cap H^{2,2}(\Omega)$ if the assumptions of Theorem 3.2 are valid. Now, if we assume further that the conditions (2.3) and (2.4) hold, we can construct upper barriers $\delta^{+}$in exactly the same way as in $\S 2$, where in the present case the barriers also satisfy $\psi \leqq \delta^{+}$, and thus we conclude

$$
\bar{u}_{\lambda} \leqq \varphi \quad \text { on } \partial \Omega
$$

for appropriate Lagrange multipliers $\lambda$.

We then take

$$
V_{0}=\int_{\Omega}\left(\bar{u}_{\lambda}-\psi\right) .
$$

The result now follows from the observation that $\bar{u}_{\lambda}$ is a solution to the variational problem (3.1) with volume $V=V_{0}$, and that the relation $V \rightarrow u_{\lambda}$, where $u_{\lambda}$ is the corresponding solution of (3.1) with volume $V$, is nondecreasing (cf. [4; formula (3.6)]).

At the end of this section we shall prove the existence of a volume $V^{*}$, depending on the obstacle $\psi$, such that the solutions of the corresponding variational problems with volume $V>V^{*}$ lie strictly above the obstacle.

To prove the existence of $V^{*}$, we need the following lemma

Lemma 3.3. Let $u \in C^{0,1}(\Omega)$ be a solution of the equation

$$
A u+H(x, u)+\mu=0 \text { in } \Omega,
$$

where $\Omega$ is an open set in $\boldsymbol{R}^{n}$ satisfying an ISC of radius $R$, and where $H$ is monotone and sublinear. Then, if

$$
\mu \leqq \min \left(-2 \cdot c \cdot R-2 \cdot \frac{n}{R},-2 \cdot c\right),
$$

where $c$ is the constant in (3.2), we can conclude

$$
u \geqq-\frac{\mu}{2 \cdot c}-1 \text {. }
$$

Proof. Let $x_{0} \in \Omega$ be arbitrary, and let $\overline{B_{R}\left(x_{0}\right)} \subset \Omega$. For simplicity assume that $x_{0}=0$, and let $\delta_{0}$ be the upper hemisphere

$$
\delta_{0}(x):=\left(R^{2}-|x|^{2}\right)^{1 / 2},|x| \leqq R .
$$


Let

$$
\delta^{-}:=\delta_{0}-\frac{\mu}{2 c}-1
$$

Then

$$
A \delta^{-}+H\left(x, \delta^{-}\right)+\mu \leqq \frac{n}{R}+c \cdot R-\frac{\mu}{2}+\mu \leqq 0
$$

in view of (3.21).

From a simple maximum principle (cf. [4; proof of Lemma 4.1]) we deduce

$$
\delta^{-} \leqq u,
$$

hence the result, since $\Omega$ can be covered by such balls.

We note that Concus and Finn [1] where the first to use hemispheres as comparison surfaces for capillary surfaces.

To apply Lemma 3.3 , let $u^{*}$ be a solution of the free capillary problem

$$
J_{\lambda}(v)+\mu \int_{\varrho} v \longrightarrow \min \quad \forall v \in B V(\Omega),
$$

where $\mu$ is sufficiently less than zero. Then, $u^{*}$ is very large, cf. (3.22), so that $u^{*}$ is strictly larger than a given obstacle $\psi$. Moreover, $u^{*}$ is also a solution to the variational problem (3.1) with volume

$$
V^{*}=\int_{\Omega}\left(u^{*}-\psi\right)
$$

This volume will be appropriate, since the relation $V \rightarrow u_{\lambda}$, where $u_{\lambda}$ is a solution of (3.1) with volume $V$, is nondecreasing, as we mentioned earlier.

4. Final remark on the physical problem. In order to prove that the solutions $u_{\lambda}$ of the new boundary value problems actually satisfy the condition

$$
u_{i} \leqq \mathcal{P} \quad \text { on } \partial \Omega
$$

we had to suppose in $\S 2$ that the boundary of $\Omega$ satisfies the specific assumption

$$
-H(x, \varphi) \leqq(n-1) \cdot H_{n-1}(x) \quad \forall x \in \partial \Omega .
$$

This is certainly necessary in the general case, for the construction of appropriate barrier functions. But in some special 
cases, including the physically interesting ones, barrier functions can be constructed under much weaker assumptions. In the physical problem the boundary obstacle $\varphi$ is always assumed to be strictly positive (the liquid surface of the large reservoir is supposed to represent the zero level), so that we may tentatively choose $\varphi$ itself as an upper barrier function, after having extended it as a positive function to the whole domain $\Omega$. Thus, the inequality

$$
A \varphi+H(x, \varphi) \geqq 0 \quad \text { in } \Omega
$$

is to be satisfied. In the physical case, $H(x, t)=\kappa \cdot t, \kappa>0$, so that we have to satisfy

$$
A \varphi+\kappa \cdot \varphi \geqq 0 \quad \text { in } \Omega .
$$

Knowing that $\varphi$ is strictly positive, this inequality will always be fulfilled when $|A \varphi|$ is small compared to $\kappa \cdot \varphi$, e.g., for $\varphi \equiv$ const.

\section{REFERENCES}

1. P. Concus and R. Finn, On a capillary surface in a gravitational field, Acta Math., 132 (1974), 207-223.

2. C. Gerhardt, Existence and regularity of capillary surfaces, Boll. UMI, 10 (1974), 317-335.

3. - Global regularity of the solutions to the capillarity problem, Ann. Scuola norm. sup. Pisa, IV. Ser., 3 (1976), 157-175.

4. - On the capillarity problem with constant volume, Ann. Scuola norm. sup. Pisa, IV. Ser., 2 (1975), 303-320.

5 . - On the regularity of solutions to variational problems in $B V(\Omega)$, Math. Z., 149 (1976), 281-286.

6. - Boundary value problems for surfaces of prescribed mean curvature, J. Math. pures et Appl., 58 (1979), 75-109.

7. J. Serrin, The problem of Dirichlet for quasilinear elliptic equations with many independent variables, Phil. Trans. Roy. Soc. London, Ser. A, 264 (1969), 413-496.

8. L. Simon and J. Spruck, Existence and regularity of a capillary surface with prescribed contact angle, Arch. rat. Mech. Analysis, 61 (1976), 19-34.

Received November 26, 1979. During the preparation of this work the author was a member of The Institute For Advanced Study in Princeton, N. J. He wishes to express his gratitude for the hospitality of this institution.

Institut für Angewandte Mathematik and

SONDERFORSCHUNGSBEREICH 123

UNIVERSität HeIDELBERg

Im Neuenheimer Feld 294

D-6900 Heidelberg, Germany 



\section{PACIFIC JOURNAL OF MATHEMATICS}

\section{EDITORS}

DONALD BABBITT (Managing Editor)

University of California

Los Angeles, CA 90024

Hugo Rossi

University of Utah

Salt Lake City, UT 84112

C. C. MOORE and ANDREW OGG

University of California

Berkeley, CA 94720
J. DUGUNDJI

Department of Mathematics

University of Southern California

Los Angeles, CA 90007

R. FinN and J. Milgram

Stanford University

Stanford, CA 94305

\section{ASSOCIATE EDITORS}
E. F. BECKENBACH
B. H. NeumanN
F. WOLF
K. YoSHIDA

\section{SUPPORTING INSTITUTIONS}

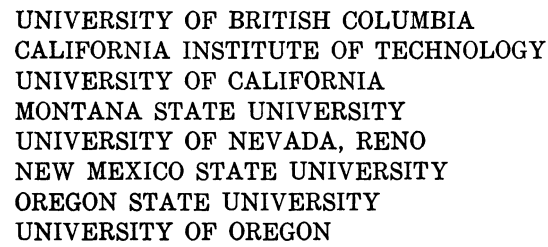

UNIVERSITY OF BRITISH COLUMBIA CALIFORNIA INSTITUTE OF TECHNOLOGY UNIVERSITY OF CALIFORNIA MONTANA STATE UNIVERSITY UNIVERSITY OF NEVADA, RENO NEW MEXICO STATE UNIVERSITY OREGON STATE UNIVERSITY UNIVERSITY OF OREGON

\author{
UNIVERSITY OF SOUTHERN CALIFORNIA \\ STANFORD UNIVERSITY \\ UNIVERSITY OF HAWAII \\ UNIVERSITY OF TOKYO \\ UNIVERSITY OF UTAH \\ WASHINGTON STATE UNIVERSITY \\ UNIVERSITY OF WASHINGTON
}

The Supporting Institutions listed above contribute to the cost of publication of this Journal, but they are not owners or publishers and have no responsibility for its content or policies.

Mathematical papers intended for publication in the Pacific Journal of Mathematics should be in typed form or offset-reproduced, (not dittoed), double spaced with large margins. Please do not use built up fractions in the text of the manuscript. However, you may use them in the displayed equations. Underline Greek letters in red, German in green, and script in blue. The first paragraph or two must be capable of being used separately as a synopsis of the entire paper. Please propose a heading for the odd numbered pages of less than 35 characters. Manuscripts, in triplicate, may be sent to any one of the editors. Please classify according to the scheme of Math. Reviews, Index to Vol. 39. Supply name and address of author to whom proofs should be sent. All other communications should be addressed to the managing editor, or Elaine Barth, University of California, Los Angeles, California, 90024.

50 reprints to each author are provided free for each article, only if page charges have been substantially paid. Additional copies may be obtained at cost in multiples of 50 .

The Pacific Journal of Mathematics is issued monthly as of January 1966. Regular subscription rate: $\$ 84.00$ a year (6 Vols., 12 issues). Special rato: $\$ 42.00$ a year to individual members of supporting institutions.

Subscriptions, orders for numbers issued in the last three calendar years, and changes of address shoud be sent to Pacific Journal of Mathematics, P.O. Box 969, Carmel Valley, CA 93924, U.S.A Old back numbers obtainable from Kraus Periodicals Co., Route 100, Millwood, NY 10546.

PUBLISHED BY PACIFIC JOURNAL OF MATHEMATICS, A NON-PROFIT CORPORATION

Printed at Kokusai Bunken Insatsusha (International Academic Printing Co., Ltd.). 8-8, 3-chome, Takadanobaba, Shinjuku-ku, Tokyo 160, Japan. 


\section{Pacific Journal of Mathematics \\ Vol. 88, No. $2 \quad$ April, 1980}

Reinhold Böhme, Stefan Hildebrandt and Engelbert Tausch, The two-dimensional analogue of the catenary ................. 247

Jean Ellen Taylor, Nonexistence of F-minimizing embedded disks ........ 279

Claus Gerhardt, A free boundary value problem for capillary surfaces ..... 285

Enrico Giusti, Generalized solutions for the mean curvature equation . . . . . 297

Jin-Tzu Chen, On the existence of capillary free surfaces in the absence of gravity.................................... 323

Leon M. Simon, Regularity of capillary surfaces over domains with corners.......................................... 363

Nicholas Jacob Korevaar, On the behavior of a capillary surface at a re-entrant corner.................................... 379

Henry Wente, The symmetry of sessile and pendent drops ............ 387

E. Gonzalez, Umberto Massari and I. Tamanini, Existence and regularity for the problem of a pendent liquid drop ...................... 399

Henry Wente, The stability of the axially symmetric pendent drop ........ 421

David Siegel, Height estimates for capillary surfaces . . . . . . . . . . . . 471

Bruce Edward Turkington, Height estimates for exterior problems of capillarity type ................................ 517

Robert Finn, The sessile liquid drop. I. Symmetric case .............. 541 